\title{
Omnidirectional Relay in Wireless Networks
}

\author{
Liang-Liang Xie \\ Department of Electrical and Computer Engineering \\ University of Waterloo, Waterloo, ON, Canada N2L 3G1 \\ Email: 1lxie@ece.uwaterloo.ca
}

\begin{abstract}
For wireless networks with multiple sources, an omnidirectional relay scheme is developed, where each node can help relay multiple sources in different directions. This is accomplished by the decode-and-forward relay strategy, with each relay binning the multiple messages to be transmitted, in the same spirit of network coding. Specially for the allsource all-cast problem, where each node is an independent source to be transmitted to all the other nodes, this scheme completely eliminates interference in the whole network, and the signal transmitted by any node is used by any other node. For networks with regular topologies, assuming no beamforming is to be performed, this omnidirectional scheme is capable of achieving the maximum achievable rate.
\end{abstract}

\section{INTRODUCTION}

In wireless networks, relay is a technique where a node can simultaneously exploit the signals transmitted by multiple nodes. Between the two fundamental relay strategies proposed in [1], especially, the decode-and-forward strategy makes it possible for the destination node to fully make use of the signal powers transmitted by both the source node and the relay node. This is still realizable when multiple relays are introduced to help the destination [2], and interference can be completely eliminated for arbitrarily large networks.

However, relaying becomes much more complicated when there are multiple sources in the network [3]. Unlike the case of a single source where all nodes are essentially transmitting the same information, multiple sources seem inevitably result in interference. Nevertheless, studies of the two-way relay channel [4], [5] indicate the possibility of no interference even if there are more than one sources.

In this paper, we develop an omnidirectional relay scheme for wireless networks with multiple sources, where, each node can simultaneously relay different sources in different directions. This is accomplished by binning multiple messages at each relay, as a generalization of the scheme proposed in [5]. The idea is basically the same as network coding. The superposition coding approach proposed in [4] can also be generalized. However, the coding schemes become much more involved in general networks and will not be considered here.

As an application, we are especially interested in the allsource all-cast problem, where each node is an independent source, to be sent to all the other nodes. We will show that for such problems, it is possible to completely eliminate interference in the network.

\section{OMNIDIRECTIONAL RELAY SCHEME}

First, we introduce a general omnidirectional relay scheme.
Consider a wireless network of $n$ nodes $\mathcal{N}=\{1,2, \ldots, n\}$. We introduce the concept of $k$-hop neighbors in the network in the following way. First, for each node $i$, define a set of nodes in its neighborhood as its 1-hop neighbors, and denote the set as $\mathcal{N}_{i}(1)$. The way of defining 1-hop neighbors depends on the network topology and will be specified later on for different networks. If node $j$ is a 1 -hop neighbor of node $i$, it is said that $j$ can reach $i$ in one hop. If furthermore, $i$ is a 1-hop neighbor of node $l$, then it is said that $j$ can reach $l$ in two hops. Similarly, it can be said that a node can reach another node in $k$ hops, for any positive integer $k$. Now, for each node $i$, its $k$-hop neighbors is defined as the set of nodes which can reach it in $k$ hops, but not in less hops, and denote this set as $\mathcal{N}_{i}(k)$. Mathematically, $\mathcal{N}_{i}(k)$ can be sequentially defined as

$$
\begin{aligned}
\mathcal{N}_{i}(k)=\left\{j: j \in \mathcal{N}_{l}(1) \text { for some } l \in \mathcal{N}_{i}(k-1),\right. \\
\text { and } \left.j \notin\{i\} \cup \mathcal{N}_{i}(1) \cup \cdots \cup \mathcal{N}_{i}(k-1)\right\} .
\end{aligned}
$$

We use block Markov coding. In block 1, each node $i$ transmits its own message $w_{i}(1)$. At the end of block 1, each node $i$ decodes at least the messages sent by its 1-hop neighbors $\left\{w_{j}(1), j \in \mathcal{N}_{i}(1)\right\}$. (It could decode more.) In block 2 , each node $i$ transmits $\left(w_{i}(2), w_{\mathcal{N}_{i}(1)}(1)\right)$ using the binning technique, where for simplicity, $w_{\mathcal{N}_{i}(1)}(1)$ stands for $\left\{w_{j}(1): j \in \mathcal{N}_{i}(1)\right\}$. At the end of block 2 , each node $i$ decodes at least the messages of its 1-hop neighbors in block 2 and the messages of its 2-hop neighbors in block 1, i.e., $\left(w_{\mathcal{N}_{i}(1)}(2), w_{\mathcal{N}_{i}(2)}(1)\right)$. In block 3 , each node $i$ transmits $\left(w_{i}(3), w_{\mathcal{N}_{i}(1)}(2), w_{\mathcal{N}_{i}(2)}(1)\right)$ using the binning technique. Generally, in block $b$, each node $i$ transmits $\left(w_{i}(b), w_{\mathcal{N}_{i}(1)}(b-\right.$ $\left.1), \ldots, w_{\mathcal{N}_{i}(b-1)}(1)\right)$ using the binning technique, and decodes $\left(w_{\mathcal{N}_{i}(1)}(b), \ldots, w_{\mathcal{N}_{i}(b)}(1)\right)$ at the end of block $b$, where, when the block number is large enough such that $\mathcal{N}_{i}(b)=\emptyset$, $w_{\emptyset}(l)=\emptyset$ for any $l$.

To realize the above omnidirectional relay scheme, we can use the regular encoding/sliding-window decoding with random binning at each node, as has been used in several simple networks in [5]. Note that backward decoding cannot be used here, since each node must not wait in decoding in order to help. This observation is exemplified in detail with a two-way multi-relay network in [3]. However, random binning can be replaced by deterministic binning that is easier to implement, although random binning is simpler to describe in the achievability proof.

For any network, obviously, the above omnidirectional relay scheme can work if the rates are small enough. However, our 
interest lies in finding network topologies where omnidirectional relay is really beneficial.

In this paper, let us consider the following AWGN wireless network channel model:

$$
Y_{j}(t)=\sum_{\substack{i \in \mathcal{N} \\ i \neq j}} g_{i, j} X_{i}(t)+Z_{j}(t), \quad \forall j \in \mathcal{N}, \quad t=1,2, \ldots
$$

where, $X_{i}(t) \in \mathbb{C}^{1}$ and $Y_{i}(t) \in \mathbb{C}^{1}$ respectively denote the signals sent and received by Node $i \in \mathcal{N} ;\left\{g_{i, j} \in \mathbb{C}^{1}: i \neq j\right\}$ denote the signal attenuation gains; and $Z_{i}(t)$ are zero-mean complex Gaussian noise with variance $N$.

We make the following assumption on the magnitude of the gains:

$$
\left|g_{i, j}\right|=g\left(d_{i, j}\right),
$$

where $d_{i, j}$ is the distance between node $i$ and node $j$, and $g(\cdot)$ is an arbitrary non-increasing function. That is, we assume that the magnitude of the gains only depends on the distance, and is non-increasing as the distance increases.

Specially, we are interested in the achievability of the following common rate for the all-source all-cast problem by the above omnidirectional relay scheme:

$$
R<\frac{1}{n-1} \log \left(1+\frac{\min _{j} \sum_{i \neq j}\left|g_{i, j}\right|^{2} P}{N}\right)
$$

where, $P$ is the individual transmit power constraint at each node. Obviously, $\sum_{i \neq j}\left|g_{i, j}\right|^{2} P$ is the total received power at node $j$ if no beamforming is performed, i.e., all the nodes are using independent codebooks. Then, $\min _{j} \sum_{i \neq j}\left|g_{i, j}\right|^{2} P$ corresponds to the node which receives the least total power. Since every node needs to decode all the other $n-1$ sources, (3) immediately gives an upper bound on the common achievable rate $R$ for the all-source all-cast problem according to the Shannon formula.

It may be possible to achieve higher rates than (3) by using correlated codebooks at different nodes, say, by beamforming. An approach is the superposition coding of multiple messages at the relay proposed in [4] (see [6] for the right formula). However, cooperating signals must represent common information in order to cooperate, which means that they cannot help the transmission of other different messages. This may not be a good choice for the all-source all-cast problem, where the messages to be transmitted by any two nodes are not completely the same.

\section{OnE-Dimensional Regular Networks}

Due to limited space, we focus our discussion on onedimensional regular networks in this paper.

Consider a wireless network of $n$ nodes located on a straight line, with an equal separation distance $d_{0}$ between any two neighboring nodes as depicted in Figure 1.

Since by the assumption (2), power attenuation only depends on the distance, it is convenient to define

$$
P_{k}=\left|g\left(k d_{0}\right)\right|^{2} P, \quad \text { for } k=1, \ldots, n-1 .
$$

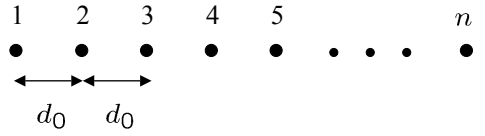

Fig. 1. A one-dimensional regular network.

Obviously, when node $i$ is transmitting at power $P$, the corresponding received power at node $j$ is $P_{|i-j|}$.

Due to the monotonicity of the gain function $g(\cdot)$, we have $P_{1} \geq P_{2} \geq \cdots \geq P_{n-1}$.

For any node $i \notin\{1, n\}$, let its 1-hop neighbors be $\mathcal{N}_{i}(1)=$ $\{i-1, i+1\}$. Let $\mathcal{N}_{1}(1)=\{2\}$ and $\mathcal{N}_{n}(1)=\{n-1\}$. We will show that the above omnidirectional relay scheme works for this one-dimensional regular network as stated in the following theorem.

Theorem 3.1: For the one-dimensional regular wireless network, with the omnidirectional relay scheme, the following common rate is achievable for the all-source all-cast problem:

$$
R<\frac{1}{n-1} \log \left(1+\frac{P_{1}+\cdots+P_{n-1}}{N}\right) .
$$

Obviously, $P_{1}+\cdots+P_{n-1}$ is the total power received by the boundary nodes 1 or 2 , which according to the topology, receive the least.

In order to prove that the omnidirectional relay scheme works for this topology at the rate (4), essentially, we need to ensure that the decoding at every node is successful, i.e., at the end of each block $b$, every node can decode $w(b)$ of its 1-hop neighbors, $w(b-1)$ of its 2-hop neighbors, and so on. If the decoding is successful up to the current block, the encoding for the next block can always be carried out. Hence, we can recursively prove the success of the decoding at each block, based on the assumption of the success of all the previous blocks.

First, we show that the decoding is successful at the boundary nodes 1 and $n$. These two nodes are unique in the way that to them, all information comes from one direction.

\section{A. Unidirectional}

Due to symmetry, we only need to address node 1 in the following.

Basically, we need to prove the condition:

C1) By the end of block $b$, node 1 can decode $w(b)$ of node $2, w(b-1)$ of node $3, \ldots, w(b-n+2)$ of node $n$.

Consider the following type of inequalities:

$$
i R<\log \left(1+\frac{P_{1}+\cdots+P_{i}}{I_{i+1}+N}\right)
$$


where $I_{i+1}:=P_{i+1}+\cdots+P_{n-1}$. Assume $1 \leq i_{1}<i_{2}<$ $\cdots<i_{m}=n-1$ is the sequence of $i \in\{1,2, \ldots, n-1\}$ for which the inequality (5) holds, where (5) at least holds for $i=n-1$ due to (4).

Define nodes $\left\{2,3, \ldots, i_{1}+1\right\}$ as Group 1; nodes $\left\{i_{1}+\right.$ $\left.2, \ldots, i_{2}+1\right\}$ as Group $2 ; \ldots$, nodes $\left\{i_{m-1}+2, \ldots, i_{m}+1\right\}$ as Group $m$.

Actually, we will show that at the end of block $b$, node 1 can decode $w(b)$ of the nodes in Group 1, w(b-1) of the nodes in Group 2, $\ldots, w(b-m+1)$ of the nodes in Group $m$. This certainly implies that the condition $\mathrm{C} 1$ ) is satisfied.

In block 1 , every node transmits its own message $w(1)$. We will show that at the end of block 1 , node 1 can decode $w(1)$ of the nodes in Group 1, due to the following inequalities:

$$
\begin{aligned}
R & \geq \log \left(1+\frac{P_{1}}{I_{2}+N}\right) \\
2 R & \geq \log \left(1+\frac{P_{1}+P_{2}}{I_{3}+N}\right) \\
& \vdots \\
\left(i_{1}-1\right) R & \geq \log \left(1+\frac{P_{1}+\cdots+P_{i_{1}-1}}{I_{i_{1}}+N}\right) \\
i_{1} R & <\log \left(1+\frac{P_{1}+\cdots+P_{i_{1}}}{I_{i_{1}+1}+N}\right)
\end{aligned}
$$

which follow from (5). First, taking the difference between the last inequality and any of the previous ones in (6)-(9), we have

$$
\begin{aligned}
R & <\log \left(1+\frac{P_{i_{1}}}{I_{i_{1}+1}+N}\right) \\
2 R & <\log \left(1+\frac{P_{i_{1}-1}+P_{i_{1}}}{I_{i_{1}+1}+N}\right) \\
& \vdots \\
i_{1} R & <\log \left(1+\frac{P_{1}+\cdots+P_{i_{1}}}{I_{i_{1}+1}+N}\right)
\end{aligned}
$$

Noticing that $P_{1} \geq P_{2} \geq \cdots \geq P_{i_{1}}$, according to the multipleaccess region, by (10)-(12), node 1 can decode $w(1)$ of the nodes in Group 1.

Now, in block 2, assuming all the nodes have successfully decoded in block 1 , each node will send $w(2)$ of itself together with $w(1)$ of its 1-hop neighbors. Especially, note that node $i_{1}+1$ will help $w(1)$ of node $i_{1}+2$. We will show that at the end of block 2, node 1 can decode $w(2)$ of Group 1, and $w(1)$ of Group 2, due to the inequalities (6)-(9) and the following inequalities:

$$
\begin{aligned}
\left(i_{1}+1\right) R & \geq \log \left(1+\frac{P_{1}+\cdots+P_{i_{1}+1}}{I_{i_{1}+2}+N}\right) \\
& \vdots \\
\left(i_{2}-1\right) R & \geq \log \left(1+\frac{P_{1}+\cdots+P_{i_{2}-1}}{I_{i_{2}}+N}\right) \\
i_{2} R & <\log \left(1+\frac{P_{1}+\cdots+P_{i_{2}}}{I_{i_{2}+1}+N}\right)
\end{aligned}
$$

which still follow from (5). Still, taking the difference between the last inequality and any of the previous ones in (13)-(15), we have

$$
\begin{aligned}
R & <\log \left(1+\frac{P_{i_{2}}}{I_{i_{2}+1}+N}\right) \\
2 R & <\log \left(1+\frac{P_{i_{2}-1}+P_{i_{2}}}{I_{i_{2}+1}+N}\right) \\
& \vdots \\
\left(i_{2}-i_{1}-1\right) R & <\log \left(1+\frac{P_{i_{1}+2}+\cdots+P_{i_{2}}}{I_{i_{2}+1}+N}\right)
\end{aligned}
$$

Moreover, taking the difference between (15) and (8), we have

$$
\left(i_{2}-i_{1}+1\right) R<\log \left(1+\frac{P_{i_{1}}+\cdots+P_{i_{2}}}{I_{i_{2}+1}+N}\right)
$$

Now, to see that the inequalities (10)-(12) and (16)-(19) ensure that at the end of block 2, node 1 can decode $w(2)$ of Group 1 and $w(1)$ of Group 2, notice that this is a multiple-access channel with two blocks. In block 1 , without considering $w(1)$ of node $i_{1}+1$, the inequalities (16)-(18) ensure the multipleaccess region. With considering $w(1)$ of node $i_{1}+1$, the help of node $i_{1}$ in block 2 will be considered, which can make use of the inequality (19). Finally, nodes of Group 1 can take care of their own $w(2)$ in block 2, due to (10)-(12).

Proceeding in a similar way, we can show that generally, at the end of block $b$, node 1 can decode $w(b)$ of Group 1, $w(b-1)$ of Group 2, $, w, w(b-m+1)$ of Group $m$. This ensures the condition $\mathrm{C} 1$ ).

\section{B. Bidirectional}

Now, we consider nodes not on the boundary. For each of them, information comes from both directions.

1) Symmetric: First, we consider the center node $\frac{n+1}{2}$ in the case where $n$ is an odd number. The proof for this node is simpler due to that the traffics on both sides of this node are symmetric.

Denote $n_{c}=\frac{n+1}{2}$. Basically, we need to prove the condition:

C2) By the end of block $b$, node $n_{c}$ can decode $w(b)$ of nodes $n_{c} \pm 1, w(b-1)$ of nodes $n_{c} \pm 2, \ldots$, $w\left(b-n_{c}+2\right)$ of nodes $\{1, n\}$.

On either side of node $n_{c}$, the powers received can be ordered as $P_{1} \geq P_{2} \geq \cdots \geq P_{n_{c}-1}$. Similarly to (5), now we consider the following type of inequalities:

$$
2 i R<\log \left(1+\frac{2 P_{1}+\cdots+2 P_{i}}{I_{i+1}^{\prime}+N}\right)
$$

where $I_{i+1}^{\prime}:=2 P_{i+1}+\cdots+2 P_{n_{c}-1}$. Assume $1 \leq i_{1}<i_{2}<$ $\cdots<i_{m}=n_{c}-1$ is the sequence of $i \in\left\{1,2, \ldots, n_{c}-1\right\}$ for which the inequality (20) holds, where (20) holds for $i=$ $n_{c}-1$ due to (4).

Define nodes $\left\{n_{c} \pm 1, \ldots, n_{c} \pm i_{1}\right\}$ as Group 1; nodes $\left\{n_{c} \pm\right.$ $\left.\left(i_{1}+1\right), \ldots, n_{c} \pm i_{2}\right\}$ as Group $2 ; \ldots$, nodes $\left\{n_{c} \pm\left(i_{m-1}+\right.\right.$ $\left.1), \ldots, n_{c} \pm i_{m}\right\}$ as Group $m$. 
We will show that at the end of block $b$, node $n_{c}$ can decode $w(b)$ of the nodes in Group 1, w(b-1) of the nodes in Group $2, \ldots, w(b-m+1)$ of the nodes in Group $m$. This certainly implies that the condition $\mathrm{C} 2$ ) is satisfied.

By (20), similarly to (10)-(12), we have

$$
\begin{aligned}
2 R & <\log \left(1+\frac{2 P_{i_{1}}}{I_{i_{1}+1}^{\prime}+N}\right) \\
4 R & <\log \left(1+\frac{2 P_{i_{1}-1}+2 P_{i_{1}}}{I_{i_{1}+1}^{\prime}+N}\right) \\
& \vdots \\
2 i_{1} R & <\log \left(1+\frac{2 P_{1}+\cdots+2 P_{i_{1}}}{I_{i_{1}+1}^{\prime}+N}\right)
\end{aligned}
$$

which, due to the property of the logarithmic function, also imply that

$$
\begin{aligned}
R & <\log \left(1+\frac{P_{i_{1}}}{I_{i_{1}+1}^{\prime}+N}\right) \\
3 R & <\log \left(1+\frac{P_{i_{1}-1}+2 P_{i_{1}}}{I_{i_{1}+1}^{\prime}+N}\right) \\
& \vdots \\
\left(2 i_{1}-1\right) R & <\log \left(1+\frac{P_{1}+2 P_{2}+\cdots+2 P_{i_{1}}}{I_{i_{1}+1}^{\prime}+N}\right)
\end{aligned}
$$

Hence, by (21)-(26), node $n_{c}$ can decode $w(1)$ of Group 1 at the end of block 1 .

Again, by (20), similarly to (16)-(19), we have

$$
\begin{aligned}
2 R & <\log \left(1+\frac{2 P_{i_{2}}}{I_{i_{2}+1}^{\prime}+N}\right) \\
4 R & <\log \left(1+\frac{2 P_{i_{2}-1}+2 P_{i_{2}}}{I_{i_{2}+1}^{\prime}+N}\right) \\
& \vdots \\
2\left(i_{2}-i_{1}-1\right) R & <\log \left(1+\frac{2 P_{i_{1}+2}+\cdots+2 P_{i_{2}}}{I_{i_{2}+1}^{\prime}+N}\right) \\
2\left(i_{2}-i_{1}+1\right) R & <\log \left(1+\frac{2 P_{i_{1}}+\cdots+2 P_{i_{2}}}{I_{i_{2}+1}^{\prime}+N}\right)
\end{aligned}
$$

which, again due to the property of the logarithmic function, also imply that

$$
\begin{gathered}
R<\log \left(1+\frac{P_{i_{2}}}{I_{i_{2}+1}^{\prime}+N}\right) \\
3 R<\log \left(1+\frac{P_{i_{2}-1}+2 P_{i_{2}}}{I_{i_{2}+1}^{\prime}+N}\right) \\
\vdots \\
\left(2 i_{2}-2 i_{1}-3\right) R<\log \left(1+\frac{P_{i_{1}+2}+2 P_{i_{1}+3}+\cdots+2 P_{i_{2}}}{I_{i_{2}+1}^{\prime}+N}\right) \\
2\left(i_{2}-i_{1}\right) R<\log \left(1+\frac{P_{i_{1}+1}+2 P_{i_{1}+2}+\cdots+2 P_{i_{2}}}{I_{i_{2}+1}^{\prime}+N}\right) \\
+\log \left(1+\frac{P_{i_{1}}}{I_{i_{1}+1}^{\prime}+N}\right)
\end{gathered}
$$

where, the last inequality follows from (29)-(30), and the following inequality:

$\log \left(1+\frac{P_{i_{1}}}{I_{i_{1}+1}^{\prime}+N}\right)+\log \left(1+\frac{P_{i_{1}+1}}{I_{i_{1}+2}^{\prime}+N}\right)>$
$\log \left(1+\frac{P_{i_{1}}}{P_{i_{1}}+I_{i_{1}+1}^{\prime}+N}\right)+\log \left(1+\frac{P_{i_{1}+1}}{P_{i_{1}+1}+I_{i_{1}+2}^{\prime}+N}\right)$.

Similarly, these inequalities ensure that at the end of block 2, node $n_{c}$ can decode $w(2)$ of Group 1 and $w(1)$ of Group 2 . Especially, the inequality (31) ensures that when considering $w(1)$ of nodes $n_{c} \pm\left(i_{1}+1\right)$, the helps of nodes $n_{c} \pm i_{1}$ in block 2 are enough.

Proceeding in a similar way, we can show that generally, at the end of block $b$, node $n_{c}$ can decode $w(b)$ of Group 1, $w(b-1)$ of Group 2, $, w, w(b-m+1)$ of Group $m$. This ensures the condition $\mathrm{C} 2$ ).

2) Non-Symmetric: Now, we consider the other nonboundary nodes, for which the traffics on both sides are generally non-symmetric.

Without loss of generality, consider any node $k \leq n / 2$. Basically, we need to prove the condition:

C3) By the end of block $b$, node $k$ can decode $w(b)$ of nodes $k \pm 1, \ldots, w(b-k+2)$ of nodes $\{1,2 k-1\}$, $w(b-k+1)$ of node $2 k, \ldots, w(b-n+k+1)$ of node $n$.

According to Figure 1, the received powers from left to right can be listed as $P_{k-1} \leq P_{k-2} \leq \cdots \leq P_{1}=P_{1} \geq$ $\cdots \geq P_{n-k}$.

Similarly, consider the following type of inequalities:

$$
\begin{array}{r}
2 i R<\log \left(1+\frac{2 P_{1}+\cdots+2 P_{i}}{I_{i+1}^{\prime \prime}+N}\right), \quad \text { when } i \leq k-1 ;(32) \\
(k-1+i) R< \\
\log \left(1+\frac{2 P_{1}+\cdots+2 P_{k-1}+P_{k}+\cdots+P_{i}}{I_{i+1}^{\prime \prime}+N}\right) \\
\text { when } k \leq i \leq n-k .
\end{array}
$$

where $I_{i+1}^{\prime \prime}:=2 P_{i+1}+\cdots+2 P_{k-1}+P_{k}+\cdots+P_{n-k}$. Assume $1 \leq i_{1}<i_{2}<\cdots<i_{m}=n-k$ is the sequence of $i$ 's for which the above inequalities hold.

Similarly define Group 1, Group 2, .., Group $m$.

If the inequality (32) doesn't hold for $i=k-1$, i.e.,

$$
2(k-1) R \geq \log \left(1+\frac{2 P_{1}+\cdots+2 P_{k-1}}{I_{k}^{\prime \prime}+N}\right),
$$

then, we have

$$
[n-1-2(k-1)] R<\log \left(1+\frac{P_{k}+\cdots+P_{n-k}}{N}\right),
$$

which means that the extra nodes in the long side (i.e., the right side in the case that $k \leq n / 2$ ) can support themselves. Then, similar to the above, at the end of block $b$, node $k$ can decode $w(b)$ of Group 1, $w(b-1)$ of Group 2, ., w $w(b-m+1)$ of Group $m$.

Now, we only need to consider the case where the inequality (32) holds for $i=k-1$. Suppose $i_{j}=k-1$. Then at the 
end of block $j$, node $k$ decode $w(j)$ of Group $1, \ldots, w(1)$ of Group $j$.

To proceed further, now the decoding process runs into non-symmetric situations. Here, the key difference from the symmetric situations is that the long side has more sources to support by itself. If the long side can still decode group by group, then there is no problem, and everything is as before.

Now, suppose at the end of block $j+1$, node $k$ cannot decode $w(1)$ of Group $j+1$, which means

$$
\begin{gathered}
\left(i_{j+1}-i_{j}+1\right) R \geq \log \left(1+\frac{P_{k}+\cdots+P_{i_{j+1}}}{I_{i_{j+1}+1}^{\prime \prime}+N}\right) \\
+\log \left(1+\frac{P_{k-1}}{P_{k}+\cdots+P_{i_{j+1}}+I_{i_{j+1}+1}^{\prime \prime}+N}\right) \\
=\log \left(1+\frac{P_{k-1}+P_{k}+\cdots+P_{i_{j+1}}}{I_{i_{j+1}+1}^{\prime \prime}+N}\right) .
\end{gathered}
$$

Here, we consider the case that nodes 1 and $2 k-1$ didn't need help themselves, i.e.,

$$
2(k-2) R \geq \log \left(1+\frac{2 P_{1}+\cdots+2 P_{k-2}}{I_{k-1}^{\prime \prime}+N}\right) .
$$

Hence, by (33) with $i=i_{j+1}$,

$\left[i_{j+1}-(k-1)+2\right] R<\log \left(1+\frac{2 P_{k-1}+P_{k}+\cdots+P_{i_{j+1}}}{I_{i_{j+1}+1}^{\prime \prime}+N}\right)$.

Taking the difference between (34) and (35), and noting that $i_{j}=k-1$,

$$
R<\log \left(1+\frac{P_{k-1}}{P_{k-1}+P_{k}+\cdots+P_{i_{j+1}}+I_{i_{j+1}+1}^{\prime \prime}+N}\right),
$$

which means that the message of node 1 can still be decoded even if that of node $2 k-1$ cannot. Similarly, messages of all the previous nodes can be decoded.

In the next block, the long side gets another helper node $2 k-2$ according to the encoding scheme. Still if

$$
\begin{aligned}
\left(i_{j+1}-i_{j}+2\right) R & \geq \\
& \log \left(1+\frac{P_{k-2}+P_{k-1}+P_{k}+\cdots+P_{i_{j+1}}}{I_{i_{j+1}+1}^{\prime \prime}+N}\right)
\end{aligned}
$$

it is not sufficient to decode messages of nodes $2 k-2$ and $2 k-1$. However, the short side can still be completely decoded with the same reasoning.

For example, consider the case that nodes 2 and $2 k-2$ didn't need help themselves, i.e.,

$$
2(k-3) R \geq \log \left(1+\frac{2 P_{1}+\cdots+2 P_{k-3}}{I_{k-2}^{\prime \prime}+N}\right) .
$$

Then, similar to (35),

$$
\begin{aligned}
& {\left[i_{j+1}-(k-1)+4\right] R<} \\
& \quad \log \left(1+\frac{2 P_{k-2}+2 P_{k-1}+P_{k}+\cdots+P_{i_{j+1}}}{I_{i_{j+1}+1}^{\prime \prime}+N}\right)
\end{aligned}
$$

Similarly, taking the difference between (36) and (37),

$2 R<\log \left(1+\frac{P_{k-2}+P_{k-1}}{P_{k-2}+P_{k-1}+\cdots+P_{i_{j+1}}+I_{i_{j+1}+1}^{\prime \prime}+N}\right)$

which (together with (35)-(37) applied to $P_{k-1}$ ) means that the messages of nodes 1 and 2 can still be decoded even if those of nodes $2 k-2$ and $2 k-1$ cannot.

The long side continues moving backward until a node where the long side can support itself. (This must happen, since without interference from the short side, the long side can always support itself due to (4).) Then the long side continues to move to the right. Now we can completely ignore the short side, and consider only the long side with some nodes knowing their neighbors. The situation is similar to the unidirectional case except that it starts with some nodes already obtaining the messages of their neighbors.

The key observation in the arguments about the nonsymmetric case is that the short side can always be decoded and then the long side can be considered separately. With this clarified, we omit the lengthy details corresponding to different subcases.

\section{Discussions}

More specific assumptions on the power gains are needed when trying to achieve (3) for networks with irregular topologies. Generally, larger networks can tolerate higher irregularities.

Although the all-source all-cast problem may not be a typical situation in practice, it can be used sometimes as an intermediate step for other applications. For example, it is the situation when the nodes in a local cluster want to exchange their quantized observations, after they, together as a virtual multiple-antenna receiver, received signals simultaneously transmitted by their respective sources.

It should be noted that the omnidirectional relay scheme is not restricted to the all-source all-cast problem, and it can also be applied to networks where not all the nodes are sources. In such cases, the common achievable rate can be higher than (3); however, it is not by simply replacing $n-1$ with the number of sources.

\section{REFERENCES}

[1] T. Cover and A. El Gamal, "Capacity theorems for the relay channel," IEEE Trans. Inform. Theory, vol. 25, pp. 572-584, 1979.

[2] L.-L. Xie and P. R. Kumar, "A network information theory for wireless communication: scaling laws and optimal operation," IEEE Trans. Inform. Theory, vol. 50, pp. 748-767, May 2004.

[3] L.-L. Xie and P. R. Kumar, " Multi-source, multi-destination, multi-relay wireless networks," IEEE Transactions on Information Theory, Special Issue on Models, Theory and Codes for Relaying and Cooperation in Communication Networks, vol. 53, pp. 3586-3595, October 2007.

[4] B. Rankov and A. Wittneben, "Achievable Rate Regions for the Twoway Relay Channel," Proc. IEEE Int. Symposium on Information Theory (ISIT), Seattle, USA, July 2006.

[5] L.-L. Xie, "Network Coding and Random Binning for Multi-User Channels," Proc. IEEE Canadian Workshop on Information Theory (CWIT), Edmonton, Canada, June 2007.

[6] J. Ponniah and L.-L. Xie, "An achievable rate region for the two-way two-relay channel," Proc. IEEE Int. Symposium on Information Theory (ISIT), Toronto, Canada, July 2008. 